\title{
Latent systemic lupus erythematosus presenting as acute ascending paralysis: a case report
}

Peiris $\mathrm{A}^{1}$ Egodage UK ${ }^{2}$ Uluwattage $\mathrm{W}^{3}$

\begin{abstract}
Here I report a 29-year old woman who presented with acute ascending paralysis and required mechanical ventilation due to severe hypokalaemia. She was found to have normal anion gap metabolic acidosis due to distal renal tubular acidosis. She had American College of Rheumatology classification criteria compatible with diagnosis of latent Systemic Lupus Erythematosus (SLE). She was successfully treated with potassium citrate and sodium bicarbonate supplementation. The case is noteworthy as an atypical presentation of SLE.
\end{abstract}

\section{Introduction}

Hypokalaemia is a recognized cause of acute paralysis. Normal anion gap metabolic acidosis secondary to distal renal tubular acidosis leads to severe hypokalaemia. Defective renal acid- base transporters are described in renal tubular defects. Connective tissue disorders can cause secondary distal renal tubular acidosis. Here we report on a rare presentation of latent SLE with acute ascending paralysis due to severe hypokaelemia secondary to distal renal tubular acidosis.

\section{Case presentation}

A 29-year-old mother of an infant presented to us with ascending paralysis for three days. She has developed lower limb paralysis initially and progressed to involve the upper limbs. On admission she had difficulty in breathing. She gave a history of lower respiratory tract infection a month ago. She has never had previous episodes of paralysis. This episode has not been associated with exercises or meals. She has had recurrent generalized tonic clonic seizures at the age of 21 years which responded well to antiepileptics. She had noticed excessive hair loss but had no oral ulcers, photosensitivity, facial rashes or joint pains. She had dry mouth but had no dry eyes nor vaginal dryness causing dyspareunia. She had no family history of muscle paralysis.

1.Postgraduate Traine in Medicine

2.Senior Registrar in General Medicine

3.Consultant Physician Teaching HospitalKarapitiya

Corresponding Author:

Anushka Peiris

peiris.anushka88@gmail.com

(D) https://orcid.org/0000-0002-2894-1828
On admission, she was dyspneic and afebrile. Her vital capacity was reduced to $4 \mathrm{~mL} / \mathrm{kg}$. All the peripheral reflexes were absent. Bilateral plantar reflexes were flexor. She had proximal muscle weakness of power grade two in both upper and lower limbs. Optic fundi were normal. The cranial nerves examination was normal. There were no cerebellar signs. She had no sensory impairment. Bladder and bowel were continent. She needed mechanical ventilator support on the first day of admission.

She had severe hypokalaemia (serum potassium $1.1 \mathrm{mmol} / \mathrm{L}$, with normal anion gap metabolic acidosis (Arterial Blood Gas pH 7.12, calculated anion gap=10). Corrected serum ionized calcium was 2.31 $\mathrm{mmol} / \mathrm{L}$. Serum Magnesium was $1.24 \mathrm{mg} / \mathrm{dL}$. Urine $\mathrm{pH}$ was 7.2. Urine for sugar was nil. Urine sodium was $71 \mathrm{mmol} / \mathrm{L}$. Urine potassium was $15 \mathrm{mmol} / \mathrm{L}$. Urine calcium to creatinine ratio was $0.09 \mathrm{mg} / \mathrm{dL}$. Urine protein to creatinine ratio was $241.4 \mathrm{~g} / \mathrm{g}$ revealing sub-nephrotic range proteinuria. Her serum creatinine was 67 micromol/L. Ultra sound examination and plain radiography revealed no evidence of nephrocalcinosis.

Her hemoglobin was $13.4 \mathrm{~g} / \mathrm{dL}$ with blood picture revealing normocytic normochromic anaemia. Direct Coomb's test was positive for Ig G and C3d.Creatinine kinase (612 U/L) was elevated.Her ESR was $129 \mathrm{~mm} /$ 1 st hour and C-reactive protein level was $8 \mathrm{mg} / \mathrm{L}$. ANA titer was $>1: 2560$ with anti-dsDNA negativity. Serum C3 was $134 \mathrm{mg} / \mathrm{dL}$ but C4 was $11 \mathrm{mg} / \mathrm{dL}$. Rheumatoid factor was $367.3 \mathrm{IU} / \mathrm{mL}$. Anti- Ro and anti-La antibodies were positive. Anti- U1RNP was $4.9 \mathrm{U} / \mathrm{mL}$. Tear break test was normal (right eye70 seconds, left eye 90 seconds).TSH 3rd generation was $1.927 \mathrm{ulU} / \mathrm{mL}$.

Cerebral Spinal Fluid analysis done at day 10 of illness onset did not reveal cytoprotein dissociation. Nerve 
conduction studies were normal except for mild $\mathrm{F}$ waves abnormalities.

Her acute condition was treated with intravenous and oral potassium and acidosis correction with sodium bicarbonate and potassium citrate. On second day she has regained her reflexes and could wean off from ventilator, but muscle power was poor. She was successfully mobilized and discharged on oral sodium bicarbonate and potassium citrate after 10 days.

Since this patient fulfilled the SLICC criteria for systemic lupus erythematosus including adult onset recurrent seizures and sub-nephrotic range proteinuria under clinical criteria, and positive ANA titer (>1:2560), and direct Coomb's test positivity under immunological criteria we concluded this case as SLE initially presenting as acute ascending paralysis due to hypokalaemia secondary to distal renal tubular acidosis.

\section{Discussion}

Systemic lupus erythematosus (SLE) is a chronic autoimmune disorder of unknown aetiology which may affect any organ of the body. SLE patients presents with variable clinical features. Some SLE patients presents with single organ involvement and they may subsequently develop the characteristic multisystem features of SLE over a period of months to years. ${ }^{1,2}$ SLE first presenting as hypokalaemic paralysis due to distal renal tubular acidosis (dRTA) is very rare.

dRTA is characterized by hypokalaemia, normal anion gap metabolic acidosis with inability to lower the urinary $\mathrm{pH}$ below 5.5 with acidemia nephrocalcinosis and nephrolithiasis.

This patient developed paralysis due to severe hypokalaemia secondary to dRTA. Urinary $\mathrm{K}+$ loss of $>$ $20 \mathrm{mmol} /$ day with hypokalaemia is suggestive of renal loss of potassium. Comparing to hypokaelemia secondary to $\mathrm{K}+$ shift into the cells, excessive renal or gastrointestinal loss of potassium needs higher doses of $\mathrm{K}+$ replacement.

Secondary dRTA occurs in autoimmune connective tissue (CT) diseases like SLE, Sjogren's and rheumatoid arthritis.3In literature mutations in genes encoding renal acid-base transporters have been suggested in the pathophysiology including mainly luminal membrane $\mathrm{H}+-\mathrm{K}+$ ATPase transporters. ${ }^{3,4} \mathrm{dRTA}$ in $\mathrm{CT}$ diseases is described in literature as due to interstitial nephritis due to mononuclear cell infiltration, deposition of antigen-antibody complexes along tubular basement membrane and antibody formation against tubular basement membrane. ${ }^{5}$

Since this patient fulfilled the ACR criteria for latent
SLE and she may manifest other multisystemic clinical features overtime, she needs to be followed up closely.

According to published literature there can be an overlap with SLE and Sjogren's with anti-Ro and anti-La antibodies positivity. ${ }^{1,4}$ Our patient had dry dyes with anti-Ro and anti-La antibodies positivity but had no other clinical features in mouth with negative tear break test nor typical histological findings on minor salivary gland biopsy to fulfill the diagnostic criteria for Sjogren's syndrome.

Long-term correction of hypokaelemia is important for reversal of neuromuscular impairment and prevention of renal function deterioration. It has been described that hypokalaemia predisposes deposition of C3, C5ba in the interstitium which aggravates renal interstitial damage. ${ }^{6}$ Supplementation of potassium citrate is important for nephrocalcinosis prevention.

This case illustrates the importance of investigating patients with dRTA for treatable secondary cause to minimize complications of an unraveled disease.

\section{References}

1.Ter Meulen CG, Pieters GFFM, Huysmans FTM. Flaccid paresis due to distal renal tubular acidosis preceding systemic lupus erythematosus. Neth J Med 2002; 60:29-32

2.Bagga A, Jain Y, Srivastava RN, Bhuyan UN. Renal tubular acidosis preceding systemic lupus erythematosus. PediatrNephrol 1993; 7: 735-736.

3.DeFranco PE,Haragsim L, Schmitz PG, BastaniB. Absence of vacuolar $\mathrm{H}(+)$-ATPase pump in the collecting duct of a patient with hypokalemic distal renal tubular acidosis and Sjögren's syndrome. Journal of the American Society of Nephrology: JASN,1995,6,2:295-301

4. Gregory AK, Barr W, Bansal VK, Vertuno LL, Fresco $\mathrm{R}$, Robinson J, Hano JE.Occurrence of renal tubular dysfunction in lupus nephritis. Arch Intern Med 1987; 147: 891- 895.

5. Caruana RJ, Barish CF, Buckalew VM Jr. Complete distal renal tubular acidosis in systemic lupus: Clinical and laboratory findings. Am J Kid Dis 1985; 6: 59-63.

6. Nakhoul F, Plavnic Y, Lichtig H, Better OS. Hypokalemic flaccid paralysis as the presenting symptom of autoimmune interstitial nephropathy. Isr J Med Sci 1993; 29: 300-303. 